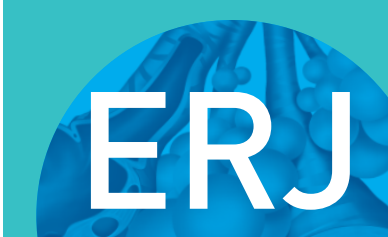

open research

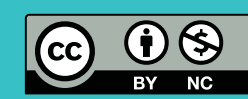

\section{Systemic biomarkers in electronic cigarette users: implications for noninvasive assessment of vaping- associated pulmonary injuries}

\author{
Kameshwar P. Singh ${ }^{1,3}$, Gina Lawyer ${ }^{1,3}$, Thivanka Muthumalage ${ }^{1}$, \\ Krishna P. Maremanda ${ }^{1}$, Naushad Ahmad Khan ${ }^{1}$, Samantha R. McDonough ${ }^{1}$, \\ Dongxia Ye ${ }^{1}$, Scott McIntosh ${ }^{2}$ and Irfan Rahman ${ }^{1}$
}

Affiliations: ${ }^{1}$ Dept of Environmental Medicine, University of Rochester Medical Center, Rochester, NY, USA ${ }^{2}$ Public Health Sciences, University of Rochester Medical Center, Rochester, NY, USA. ${ }^{3}$ These authors contributed equally to this work.

Correspondence: Irfan Rahman, Dept of Environmental Medicine, University of Rochester Medical Center, Box 850, 601 Elmwood Avenue, Rochester, NY 14642, USA. E-mail: irfan rahmandurmc.rochester.edu

\section{ABSTRACT}

Background: Electronic cigarettes (e-cigs) were introduced as electronic nicotine delivery systems, and have become very popular in the USA and globally. There is a paucity of data on systemic injury biomarkers of vaping in e-cig users that can be used as a noninvasive assessment of vaping-associated lung injuries. We hypothesised that characterisation of systemic biomarkers of inflammation, anti-inflammatory, oxidative stress, vascular and lipid mediators, growth factors, and extracellular matrix breakdown may provide information regarding the toxicity of vaping in e-cig users.

Methods: We collected various biological fluids, i.e. plasma, urine, saliva and exhaled breath condensate $(\mathrm{EBC})$, measured pulmonary function and vaping characteristics, and assessed various biomarkers in e-cig users and nonusers.

Results: The plasma samples of e-cig users showed a significant increase in biomarkers of inflammation (interleukin (IL)-1 $\beta$, IL-6, IL-8, IL-13, interferon (IFN)- $\gamma$, matrix metalloproteinase-9, intercellular cell adhesion molecule-1) and extracellular matrix breakdown (desmosine), and decreased pro-resolving lipid mediators (resolvin $\mathrm{D}_{1}$ and resolvin $\mathrm{D}_{2}$ ). There was a significant increase in growth factor (endothelial growth factor, vascular endothelial growth factor, $\beta$-nerve growth factor, platelet-derived growth factor-AA, stem cell factor, hepatocyte growth factor and placental growth factor) levels in plasma of e-cig users versus nonusers. E-cig users showed a significant increase in levels of inflammatory biomarker IFN- $\gamma$, oxidative stress biomarker 8-isoprostane and oxidative DNA damage biomarker 8-oxo-dG in urine samples, and of inflammatory biomarker IL-1 $\beta$ in saliva samples. EBC showed a slight increase in levels of triglycerides and 8-isoprostane in e-cig users compared with normal nonusers.

Conclusion: E-cig users have increased levels of biomarkers of inflammation and oxidative stress, reduced proresolving anti-inflammatory mediators, and endothelial dysfunction, which may act as risk factors for increasing susceptibility to systemic diseases. The identified noninvasive biomarkers can be used for determining e-cig vaping-associated lung injuries, and for regulatory and diagnostic aspects of vaping in humans.

@ERSpublications

E-cig use adversely affects oxidative stress and inflammatory responses, and induces tissue remodelling. The identified biomarkers can be used for assessment of vaping-associated lung injuries, and for regulatory and diagnostic aspects of vaping in humans. http://bit.ly/2nxZQ8R

Cite this article as: Singh KP, Lawyer G, Muthumalage T, et al. Systemic biomarkers in electronic cigarette users: implications for noninvasive assessment of vaping-associated pulmonary injuries. ERJ Open Res 2019; 5: 00182-2019 [https://doi.org/10.1183/23120541.00182-2019].

Received: 21 July 2019 | Accepted after revision: 3 Oct 2019

Copyright $\odot$ ERS 2019. This article is open access and distributed under the terms of the Creative Commons Attribution Non-Commercial Licence 4.0 


\section{Introduction}

Electronic cigarettes (e-cigs) have gained recent popularity in the USA and throughout the world. Despite the perception among e-cig users that e-cig vaping is safer than cigarette smoking, there are large numbers of epidemiological studies showing its effects otherwise. E-cigs are battery-operated devices that generate inhalable aerosol known as vapour. This vapour is created by heating the "e-liquid mixture" that has been filled inside the e-cig. E-cig liquid contains propylene glycol, vegetable glycerin, nicotine in various concentrations, flavouring agents and other additive compounds [1]. Recently, various other additives including terpenes, mineral oil, organic compounds and adulterated compounds have been added to e-cig juices and various vaping products. The vapour generated by e-cigs is composed of ultrafine particles which contain numerous toxic chemicals, including acetaldehyde, acrolein, toluene and formaldehyde, in lower concentrations than in cigarette smoke [2]. Increased levels of aldehydes (e.g. formaldehyde, acrolein and acetaldehyde) were found in the exhaled breath of e-cig users during vaping [3].

Flavouring chemicals present in e-cig liquid, such as diacetyl (2,3-butanedione) and 2,3-pentanedione, have been associated with bronchiolitis obliterans syndrome (popcorn lung disease) [4]. Recent studies suggest that e-cig use can change vital signs and symptoms, such as heart rate and blood pressure, in users and affect the cardiovascular system [5-9]. A single use of e-cigs can produce oxidative stress and endothelial cell dysfunction $[10,11]$. In addition, the rapid increase in circulating endothelial progenitor cells found in the blood of e-cig users was comparable to the cell numbers found in cigarette smokers, which may be a sign of acute endothelial cell dysfunction and vascular injury [12]. E-cig users have increased neutrophil activation, changes in mucin secretion and altered innate immune response in the lungs [13]. Moreover, e-cig users have impaired lung function and these changes exhibited peripheral obstructive airway involvement [14]. In a recent study, e-cig use has been found to be associated with lung inflammation through impaired systemic inflammation signalling [15]. E-cig users exposed to these harmful organic and inorganic compounds (including metals) are expected to be more susceptible to develop cancer than nonusers [16]. Furthermore, these chemical compounds in e-cigs may pose a risk of asthma $[17,18]$.

Studies on systemic biomarkers of inflammation, oxidative stress, platelet activation and systemic immune markers in cigarette smokers $[19,20]$ and systemic serum biomarkers in chronic obstructive pulmonary disease (COPD) patients are abundant [21-23]. However, there is a gap in knowledge on systemic biomarkers related to the aforementioned parameters in blood, urine, saliva and exhaled breath condensate (EBC) analysis in e-cig users. We hypothesise that detailed characterisation of various systemic noninvasive biomarkers of inflammation, oxidative stress, growth factors, vascular mediators, lipid mediators and extracellular matrix breakdown may provide information regarding molecular mechanisms through which e-cigs cause cellular/tissue damage. Hence, we determined selective biomarkers of inflammation, oxidative stress, pro-resolving mediators, triglycerides, growth factors and lung damage in plasma, urine, saliva and EBC of e-cig users and normal nonusers. We further implicated that these noninvasive markers may serve as biomarkers of vaping-associated lung injuries.

\section{Methods}

\section{Participants and procedure}

The study was conducted at the University of Rochester Medical Center (Rochester, NY, USA) (Institutional Review Board approval RSRB00064337). The study participants were recruited/enrolled at the General Clinical Research Center of the University of Rochester Medical Center through various local newspaper and magazine advertisements, word of mouth, and flyers posted in and around the university campus. The participants were selected based on a self-reported questionnaire containing information about demographic variables, e-cig vaping history and vaping behaviour.

All the potential participants identified were screened for eligibility by using the following criteria: 1) age 21-65 years, 2) normal subjects to have never used any tobacco products, 3) e-cig users to have not used other forms of tobacco products or smoking devices (e.g. cigarettes or waterpipes), 4) none of the participants to have a history of chronic disease, such as lung and heart diseases, diabetes and cancer, 5) none of the participants to have respiratory infections or be using any anti-inflammatory and/or corticosteroid drugs, and 6) female participants to not be currently breastfeeding or pregnant. Written informed consent was obtained from all study participants.

\section{Study design}

This study was planned to analyse various biomarkers in the biological samples of e-cig users. Before sample collection from each subject, body mass index (BMI) was calculated and lung function testing was performed. Collected biological samples included plasma, urine, saliva and EBC. Venous blood (20-25 mL) was collected and the plasma samples were stored immediately at $-80^{\circ} \mathrm{C}$ until analysed. Urine 
samples $(20-25 \mathrm{~mL})$ provided by participants were aliquoted and stored in a freezer at $-20^{\circ} \mathrm{C}$ until analysed. SalivaBio Swabs (Salimetrics, Newmarket, UK) were used for the collection of saliva samples from participants for various analyses. The collection procedure was followed as per the vendor's instructions. The samples were stored at $-80^{\circ} \mathrm{C}$. The participants were asked to refrain from eating, drinking and vaping for at least $3 \mathrm{~h}$ prior to $\mathrm{EBC}$ collection, and samples were obtained prior to lung function assessments. EBC samples were collected during tidal breathing for $10 \mathrm{~min}$ using an RTube collection device (Respiratory Research, Charlottesville, VA, USA). Samples were aliquoted and stored at $-80^{\circ} \mathrm{C}$.

\section{Demographics and e-cig vaping status}

The participants provided demographic information which included their age, sex and ethnicity. They also provided information regarding e-cig vaping duration, vaping frequency and vaping sessions. The study participants were divided into two groups: normal nonusers $(n=26)$ and e-cig users $(n=22)$ (table 1).

\section{Measurement of pulmonary function}

Pulmonary function tests were performed using a portable spirometer (Vitalograph micro 6300; Vitalograph, Lenexa, KS, USA). Forced expiratory volume in $1 \mathrm{~s}\left(\mathrm{FEV}_{1}\right), \mathrm{FEV}_{1} \%$ pred, forced vital capacity (FVC) \%, peak expiratory flow (PEF) \% pred and forced expiratory ratio $\left(\mathrm{FEV}_{1} / \mathrm{FVC}_{\mathrm{F}} \%\right.$ ) were recorded. The best of three attempts of the forced manoeuvre was measured.

\section{Measurement of plasma cotinine}

The plasma cotinine concentration was measured in plasma samples of participants to confirm the status of electronic nicotine delivery system/nicotine exposure by e-cigs using the Salimetrics high-sensitivity quantitative enzyme immunoassay kit (Salimetrics, Carlsbad, CA, USA) following the manufacturer's instructions.

\section{Measurement of biomarkers by multiplex panel assay}

Plasma and urine cytokine and chemokine biomarkers were assayed using the Luminex Human XL Cytokine Discovery Panel and a custom 9-plex Human Magnetic Luminex Assay (R\&D Systems, Minneapolis, MN,

TABLE 1 Baseline demographic, anthropometric and spirometry characteristics of study

participants: normal subjects and electronic cigarette (e-cig) users

\begin{tabular}{|c|c|c|}
\hline & Normal subjects ${ }^{\#}$ & E-cig users ${ }^{\#}$ \\
\hline Subjects & 26 & 22 \\
\hline \multicolumn{3}{|l|}{ Age years } \\
\hline Overall & $33.88 \pm 14.07$ & $35.54 \pm 12.21$ \\
\hline Male & $37.27 \pm 14.22$ & $32.00 \pm 14.50$ \\
\hline Female & $31.40 \pm 13.91$ & $38.50 \pm 9.58$ \\
\hline \multicolumn{3}{|l|}{ Sex } \\
\hline Male & $11(42.3)$ & 10 (45.5) \\
\hline Female & 15 (57.7) & $12(54.5)$ \\
\hline $\mathrm{BMI} \mathbf{k g} \cdot \mathrm{m}^{-2}$ & $24.86 \pm 5.06$ & $27.39 \pm 5.39$ \\
\hline \multicolumn{3}{|l|}{ Ethnicity } \\
\hline White & 18 (69.2) & $11(50.0)$ \\
\hline African-American & $3(11.5)$ & $6(27.3)$ \\
\hline Asian & $4(15.4)$ & $3(13.6)$ \\
\hline Hispanic & $1(3.9)$ & $2(9.1)$ \\
\hline \multicolumn{3}{|l|}{ E-cig vaping status } \\
\hline Vaping duration years & & $2.00 \pm 1.64$ \\
\hline Vaping frequency day ${ }^{-1}$ & & $7.75 \pm 3.14$ \\
\hline Vaping session min & & $8.79 \pm 5.50$ \\
\hline \multicolumn{3}{|l|}{ Lung function variables } \\
\hline $\mathrm{FEV}_{1} \%$ pred & $98.08 \pm 12.72$ & $91.22 \pm 10.46$ \\
\hline FVC $\%$ & $99.17 \pm 11.69$ & $95.72 \pm 13.20$ \\
\hline $\mathrm{FEV}_{1} / \mathrm{FVC} \%$ & $99.26 \pm 6.12$ & $95.50 \pm 8.98$ \\
\hline PEF \% pred & $84.56 \pm 18.48$ & $76.90 \pm 19.82$ \\
\hline \multicolumn{3}{|c|}{$\begin{array}{l}\text { Data are presented as } n \text {, mean } \pm \text { SD or } n(\%) \text {. BMI: body mass index; } F_{E V} \text { : forced expiratory volume in } 1 \mathrm{~s} ; \\
\text { FVC: forced vital capacity; PEF: peak expiratory flow. \#: not all biological samples collected from normal } \\
\text { subjects and e-cig users were used for each biomarker analysis. }\end{array}$} \\
\hline
\end{tabular}


USA). Growth factors were measured using a custom 12-plex Magnetic Luminex Assay (R\&D Systems). The plasma and urine levels of interleukin (IL)- $1 \alpha$, IL- $\beta$, IL-6, IL-8, IL-10, IL-13, IL-33, interferon (IFN)- $\gamma$, tumour necrosis factor (TNF)- $\alpha$, eotaxin, macrophage inflammatory protein (MIP)-1 $\alpha$, MIP-1 $\beta$, granulocyte colony-stimulating factor (G-CSF), granulocyte-macrophage colony-stimulating factor (GM-CSF), monocyte chemoattractant protein (MCP)-1, CXCL1, CXCL2 and RANTES were measured by the XL Cytokine Discovery Panel, while a custom 9-plex assay was used to measure S100A8, S100A9, plasminogen activator inhibitor (PAI)-1/serpine-1, matrix metalloproteinase (MMP)-9, myeloperoxidase, galectin-3, CC10/CC16, RAGE and EN-RAGE. Plasma growth factor levels of brain-derived neurotrophic factor (BDNF), bone morphogenetic protein (BMP)-2, endothelial growth factor (EGF), basic fibroblast growth factor (bFGF)/ FGF2, hepatocyte growth factor (HGF), leukaemia inhibitory factor (LIF), $\beta$-nerve growth factor (NGF), platelet-derived growth factor (PDGF)-AA, placental growth factor (PIGF), stem cell factor (SCF)/c-kit ligand, transforming growth factor (TGF)- $\alpha$ and vascular endothelial growth factor (VEGF) were included in the custom 12-plex assay. The Luminex kits were used following the manufacturer's protocols.

\section{Measurement of biomarkers by ELISA}

Plasma levels of biomarkers were measured using commercially available ELISA kits: C-reactive protein (CRP) (Sigma, St Louis, MO, USA), desmosine (MyBiosource, San Diego, CA, USA), 8-isoprostane, prostaglandin $\mathrm{E}_{2}$, thromboxane $\mathrm{B}_{2}$, resolvin $\mathrm{D}_{1}$, resolvin $\mathrm{D}_{2}$, resolvin $\mathrm{E}_{1}$, leukotriene $\mathrm{E}_{4}$ (Cayman, Ann Arbor, MI, USA), fibrinogen (Abcam, Cambridge, MA, USA), intracellular adhesion molecule (ICAM)-1 (R\&D Systems), 4-hydroxynonenal, malondialdehyde (Cell Biolabs, San Diego, CA, USA) and lipoxin $\mathrm{A}_{4}$ (TSZ ELISA, Waltham, MA, USA). Urine samples were analysed for 8 -isoprostane, leukotriene $\mathrm{E}_{4}$, desmosine and 8-oxo-dG (HT 8-Oxo-dG Human ELISA; Trevigen, Gaithersburg, MD, USA). Salivary biomarkers IL-1 IL-6, prostaglandin $E_{2}$, resolvin $D_{1}$ and resolvin $D_{2}$ were also measured. $E B C$ samples were analysed for 8 -isoprostane. All these biomarkers were measured quantitatively as per the manufacturers' instructions.

\section{Triglyceride quantification in EBC}

Triglyceride levels in EBC samples from normal nonusers and e-cig users were quantified using a fluorometric kit (BioVision, Milpitas, CA, USA) according to the manufacturer's instructions. In brief, the standards were prepared from $0-10 \mathrm{nmol}$ using $0.02 \mathrm{mM}$ triglyceride standard. $50 \mu \mathrm{L}$ EBC sample and standards were pipetted into the wells and incubated with $2 \mu \mathrm{L}$ lipase for $20 \mathrm{~min}$. Subsequently, the triglyceride reaction mix was prepared with $0.4 \mu \mathrm{L} \cdot \mathrm{well}^{-1}$ probe, enzyme mix and assay buffer. After adding $50 \mu \mathrm{L}$ reaction mix to the samples and standards, the plate was incubated for $30 \mathrm{~min}$ prior to recording the fluorescence (excitation/emission 535/590 nm). Final triglyceride concentrations were presented in millimolar units in EBC samples.

\section{Statistical analysis}

Statistical analysis was performed using Prism version 8.0.1 (GraphPad, La Jolla, CA, USA). Categorical variables data are presented as percentages and continuous variables are presented as mean \pm SEM, unless otherwise indicated. Statistical significance was determined by the t-test. A p-value $<0.05$ was considered significant.

\section{Results}

The baseline demographic, BMI, e-cig vaping status and lung function variables are summarised in table 1. The study enrolled 48 subjects, of which 22 adult e-cig users and 26 normal subjects were examined. The mean age of e-cig users was higher compared with normal subjects. BMI was higher in e-cig users compared with normal subjects. E-cig users averaged $2.00 \pm 1.64$ years of vaping for $7.75 \pm 3.14$ times per day in $8.79 \pm 5.50 \mathrm{~min}$ sessions. The majority of participants were Caucasian, followed by African-American, Asian and Hispanic. Pulmonary function test parameters such as $\mathrm{FEV}_{1}$ (\% pred), FVC (\%), $\mathrm{FEV}_{1} / \mathrm{FVC}(\%)$ and PEF (\% pred) of e-cig users did not show significant changes compared with normal subjects. We did not find any significant Pearson correlation in vaping status and vaping frequency between male and female subjects in normal subjects or e-cig users.

Plasma cotinine levels were significantly higher in e-cig users $\left(164.70 \pm 39.92 \mathrm{ng} \cdot \mathrm{mL}^{-1}\right)$ compared with normal subjects $\left(3.86 \pm 2.74 \mathrm{ng} \cdot \mathrm{mL}^{-1}\right)$, indicating all users were actively vaping e-cigs containing nicotine.

\section{Biomarkers of systemic inflammation, anti-inflammatory, oxidative stress, vascular and lipid} mediators, and extracellular matrix breakdown in plasma

Plasma levels of IL-6, IL-8, IL-13 and MMP-9 were significantly higher, whereas levels of CXCL1 and RAGE were significantly lower, in e-cig users compared with normal subjects (figure 1). The levels of IL-1 $\beta$, IL-33, TNF- $\alpha$, IFN- $\gamma$, GM-CSF, EN-RAGE, galectin-3 and myeloperoxidase showed insignificant small decreases in e-cig users compared with normal subjects. Nonsignificant changes were observed in the levels of IL- $1 \alpha$, MCP-1, MIP-1 $\alpha$, MIP-1 $\beta$, IL-10, CC10/CC16, RANTES, eotaxin, S100A8 and S100A9 
(figure 1). The plasma of e-cig users showed a nonsignificant change in levels of CXCL2 (e-cig $2603 \pm 454.8$ versus normal $3108 \pm 443.6 \mathrm{pg} \cdot \mathrm{mL}^{-1}$ ) and a significant change in levels of G-CSF (e-cig $10.86 \pm 2.03$ versus normal $\left.41.35 \pm 3.66 \mathrm{pg} \cdot \mathrm{mL}^{-1} ; \mathrm{p}<0.001\right)$ compared with normal subjects.

The biomarkers for vascular function (ICAM-1) and extracellular matrix breakdown (desmosine) were significantly higher in e-cig users compared with normal subjects. Other biomarkers, such as fibrinogen,
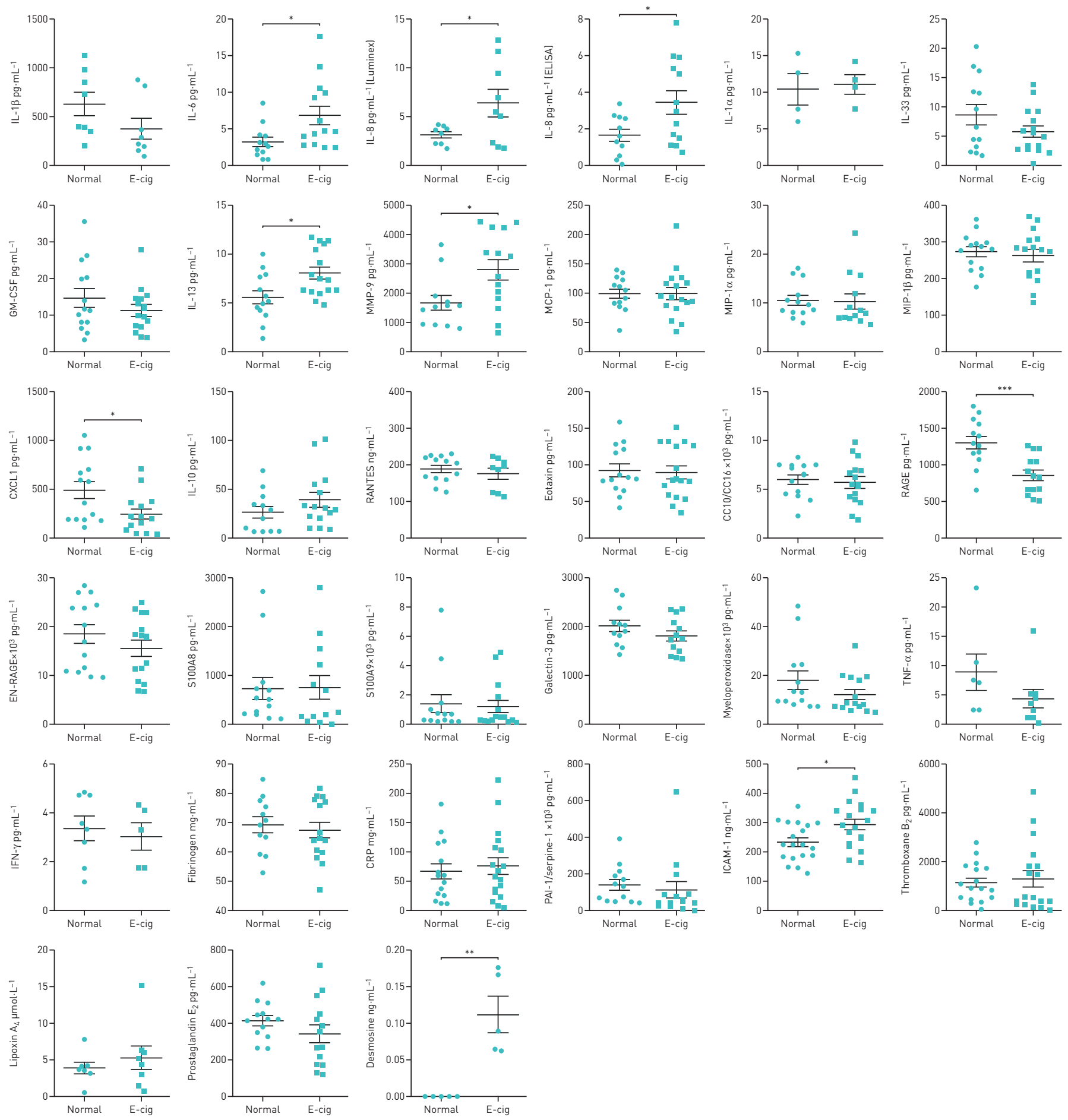

FIGURE 1 Plasma biomarkers of systemic inflammation, anti-inflammatory, vascular and lipid mediators, and extracellular matrix breakdown in normal subjects and e-cigarette (e-cig) users. IL: interleukin; GM-CSF: granulocyte-macrophage colony-stimulating factor; MMP: matrix metalloproteinase; MCP: monocyte chemoattractant protein; MIP: macrophage inflammatory protein; TNF: tumour necrosis factor; IFN: interferon; CRP: C-reactive protein; PAI: plasminogen activator inhibitor; ICAM: intracellular adhesion molecule. Data are presented as mean \pm SEM $(n=4-20) .{ }^{*}: p<0.05 ;{ }^{* *}: p<0.01 ; * *: p<0.001$. 
CRP, PAI-1/serpine-1, thromboxane $\mathrm{B}_{2}$, lipoxin $\mathrm{A}_{4}$ and prostaglandin $\mathrm{E}_{2}$, showed nonsignificant changes in e-cig users compared with nonusers (figure 1). Oxidative stress biomarkers in plasma, such as 8-isoprostane, 4-hydroxynonenal and malondialdehyde, showed no significant changes between the two groups (figure 2).

Biomarkers of lipid mediators for lung inflammation and resolution, such as pro-resolving resolvin $\mathrm{D}_{1}$ and resolvin $\mathrm{D}_{2}$, were also measured in plasma. These levels were significantly decreased in e-cig users compared with normal subjects (figure 2).

\section{Growth factors levels in plasma samples}

Plasma growth factors EGF, VEGF, $\beta$-NGF, PDGF-AA, SCF, HGF and PIGF were significantly increased in e-cig users compared with normal subjects, suggesting adverse health effects. However, the other growth factors, such as BDNF, BMP-2, TGF- $\alpha$ and bFGF/FGF2, showed no change in e-cig users compared with normal subjects (figure 3 ). The level of LIF in plasma was undetectable in both e-cig users and normal subjects.

\section{Biomarkers of systemic inflammation, vascular mediators, oxidative stress, tissue injury repair,} oxidative DNA damage and extracellular matrix breakdown in urine

Significant increases in levels of IFN- $\gamma, 8$-isoprostane and 8-oxo-dG were shown in urine samples of e-cig users compared with normal subjects. Nonsignificant increased levels of MIP-1 $\alpha$, GM-CSF, IL-33, RANTES and MMP-9, and decreased levels of IL-8, IL-10, IL-13 and S100A8, were observed in e-cig users compared with normal subjects. Other inflammatory biomarkers, such as MCP-1, eotaxin, MIP-1 $\alpha$, MIP-1 $\beta$, CC10/CC16, leukotriene $\mathrm{E}_{4}$, RAGE, EN-RAGE, S100A9 and PAI-1/serpine-1, showed no change in e-cig users compared with normal subjects (figure 4a). Urine levels of IL-1 $\alpha$, IL-1 $\beta$, IL-6, TNF- $\alpha$, G-CSF and CXCL2 were undetectable. However, no change was observed in galectin-3, myeloperoxidase and desmosine levels in urine samples of e-cig users compared with normal subjects (figure 4a).
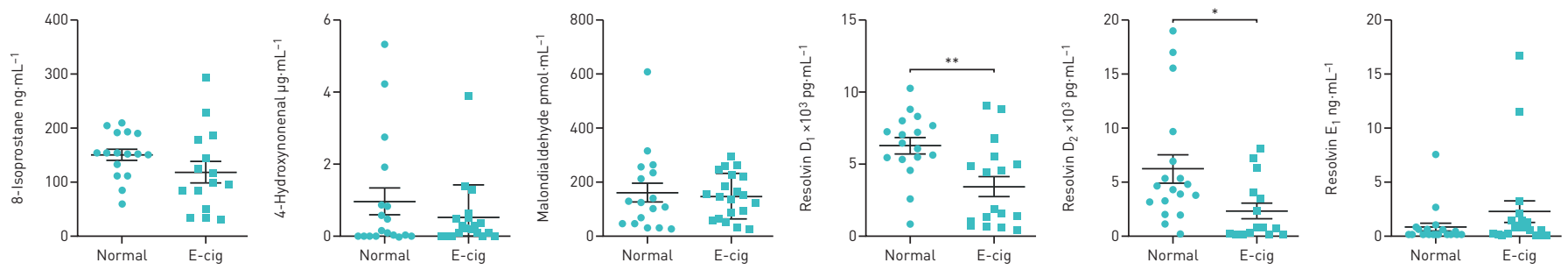

FIGURE 2 Plasma biomarkers of oxidative stress in normal subjects and e-cigarette (e-cig) users. Data are presented as mean \pm SEM ( $\mathrm{n}=15-20$ ). $*: p<0.05 ; * *: p<0.01$
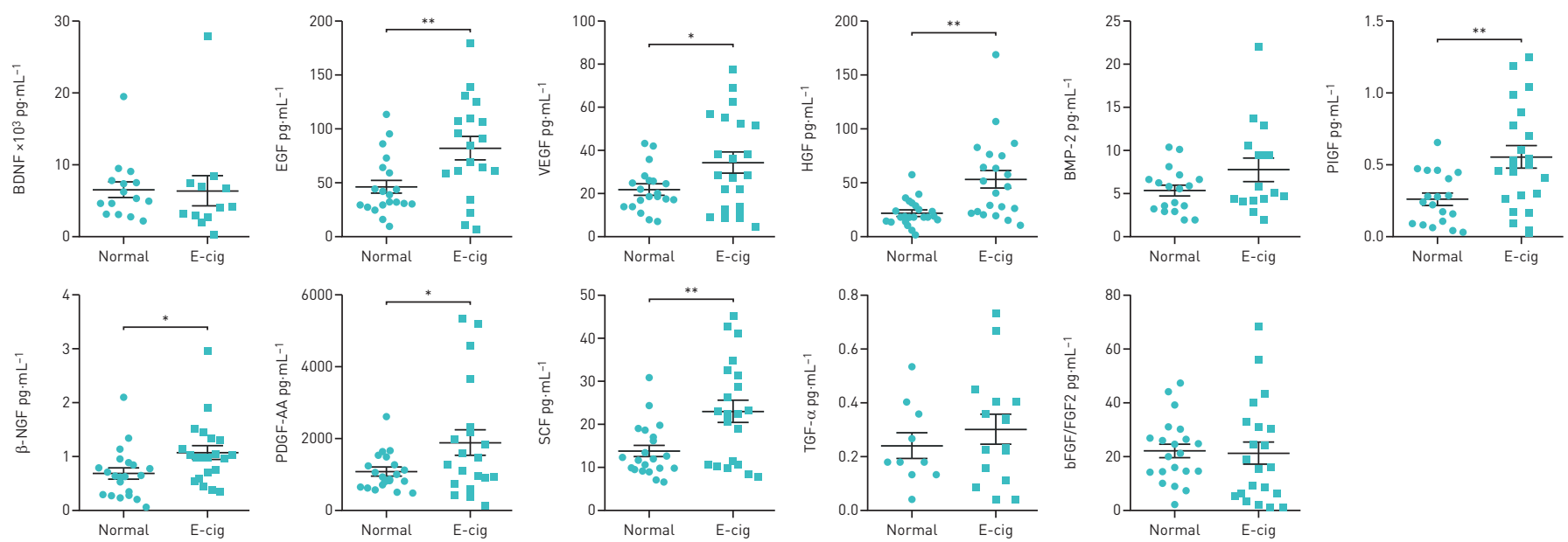

FIGURE 3 Plasma growth factors in normal subjects and e-cigarette (e-cig) users. BDNF: brain-derived neurotrophic factor; EGF: endothelial growth factor; VEGF: vascular endothelial growth factor; HGF: hepatocyte growth factor; BMP: bone morphogenetic protein; PIGF: placental growth factor; NGF: nerve growth factor; PDGF: platelet-derived growth factor; SCF: stem cell factor; TGF: transforming growth factor; bFGF: basic fibroblast growth factor. Data are presented as mean \pm SEM $(n=10-21)$. ${ }^{*}: p<0.05 ;{ }^{* *}: p<0.01$. 


\section{Biomarkers of systemic inflammation and lipid mediators in saliva}

We extended our assessment of selected biomarkers, such as IL- $1 \beta$, IL-6, prostaglandin $E_{2}$, resolvin $D_{1}$ and resolvin $\mathrm{D}_{2}$, in saliva samples of e-cig users and normal subjects. The saliva samples of e-cig users showed significant increases in IL-1 $\beta$ compared with normal subjects (figure $4 \mathrm{c}$ ). A nonsignificant increase of IL-6, resolvin $D_{2}$, and a decrease in resolvin $D_{1}$ and prostaglandin $E_{2}$, was observed in e-cig users compared with normal subjects (figure $4 \mathrm{c}$ ).
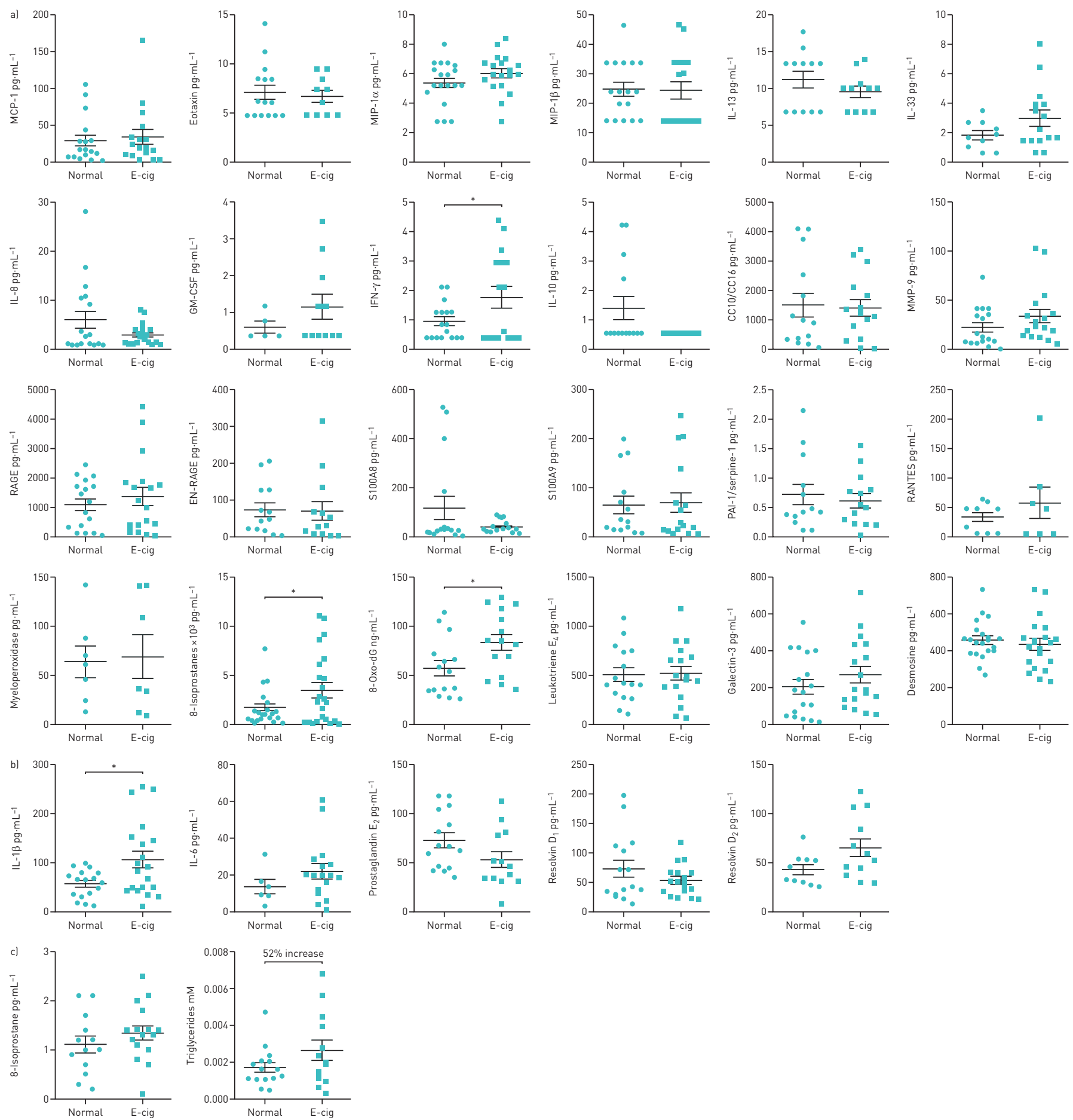

FIGURE 4 a) Urinary, b) salivary and c) exhaled breath condensate biomarkers of systemic inflammation, vascular mediators, oxidative stress, tissue injury repair, oxidative DNA damage, extracellular matrix breakdown and triglycerides in normal subjects and e-cigarette le-cigl users. MCP: monocyte chemoattractant protein; MIP: macrophage inflammatory protein; IL: interleukin; GM-CSF: granulocyte-macrophage

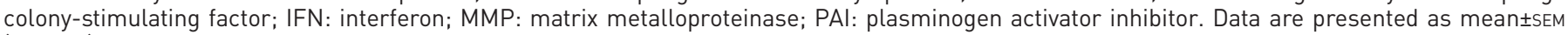
$(n=5-23) . *: p<0.05$. 
Biomarkers of oxidative stress in EBC

Biomarkers for oxidative stress (8-isoprostane) and lipids (triglycerides) were measured in EBC. There was a nonsignificant increase in the levels of 8 -isoprostane (22\% increase) and triglycerides (52\% increase) in e-cig users compared with normal subjects (figure $4 \mathrm{~d}$ ).

\section{Discussion}

We carried out a comprehensive analysis of various systemic biomarkers of inflammation, oxidative stress, growth factors, vascular mediators, lipid mediators and extracellular matrix breakdown in biological samples of e-cig users and normal subjects. We found the levels of systemic biomarkers of inflammation, endothelial dysfunction, elastin breakdown, oxidative stress, pro-resolving lipid mediators and triglycerides were altered in e-cig users in various biological fluids. Plasma cotinine levels were significantly increased in e-cig users, indicating they were exposed to nicotine during vaping e-cigs.

It is perceived that e-cig users have significantly increased levels of oxidative stress marker 8-isoprostane and oxidative DNA damage marker 8-oxo-dG. 8-isoprostane is formed in vivo by free radical peroxidation of arachidonic acid and levels in biological fluids indicate oxidative stress in vivo. Cigarette smoke induces oxidative stress and airway inflammation, which leads to COPD [23-25]. Prolonged oxidative exposure may produce various diseases, such as cardiovascular disease, lung fibrosis, blood and lung cancer [26]. Furthermore, observed increases in 8 -isoprostane (22\% increase) and triglycerides (52\% increase) in EBC suggest that oxidative stress induces alteration in epithelial lining fluid metabolites in e-cig users. Triglycerides are formed due to fatty acid (endogenous or exogenous) breakdown/oxidation, possibly due to tetrahydrocannabinol (THC) oil in some e-cigs becoming oxidised via aerosol-mediated oxidative stress. It is important to consider that some e-cig users may be vaping other products that are rich in organic oils, leading to the accumulation of lipids in macrophages (lipoid/foamy macrophages). Our data showing a modest increase in EBC triglyceride levels as a noninvasive biomarker highlight the importance of further studies, with the potential to cause acute lung injury (i.e. lipoid pneumonia by vaping-associated pulmonary disease) [27-30].

Several studies have reported that cigarette smoke can increase oxidative stress and increase the release of 8 -isoprostane in human respiratory epithelial cells [31-34]. Cigarette smoke-induced oxidative stress activates the inflammatory response by upregulating cytokines, such as IL-6 and IL-8 [35, 36]. IL-6 has been linked to inflammation, ageing, immunity and various chronic diseases $[37,38]$, whereas IL- 8 has been linked to the pathogenesis of chronic inflammation and cancer [39]. IL-13 plays a role in various inflammatory diseases, including lung fibrosis, asthma, allergy and cancer [40, 41]. IL-1 $\beta$ is a marker of inflammatory status. Another study reported similar findings of increased levels of salivary IL-1 $\beta$ in e-cig users [42]. The inflammatory mediator IFN- $\gamma$ was increased in urine samples of e-cig users compared with nonusers, which was also seen in tobacco smokers [43]. Supporting the observations in our study, pro-inflammatory effects of e-cig condensate on human alveolar macrophages in vitro have been reported in another study [44]. Similarly, primary airway smooth muscle cells from COPD patients have a greater release of inflammatory mediators compared with those of non-COPD patients, suggesting a greater risk from e-cigs in COPD patients [45].

Corroborating the increased IL-6 data in e-cig users in this study, LERNER et al. [46] demonstrated significantly increased IL-6 cytokine levels in bronchoalveolar lavage fluid (BALF) in a 3-day acute Blu e-cig side-stream aerosol C57BL/6J mouse model. In contrast, another group showed reduced IL-6 levels after a 2-week exposure to NJOY e-cig vapour in a mouse model [47]. Consistent with LERNER et al. [46], a study with C57BL/6J mice exposed to V4L CoolCart vapour for 3 days showed increased lung IL-6 mRNA levels and pulmonary oedema, with a 5.5-fold wet-to-dry ratio [48]. These outcomes may suggest that the inflammatory response depends on the frequency and the e-cig brand used. CD-1 mice exposed to e-cig aerosols for 1 month showed increased $\mathrm{KC}$ and triggering receptor expressed on myeloid cells-1 (TREM-1) pro-inflammatory mediators in BALF, bolstering the hypothesis that exposure to e-cig aerosols induces an inflammatory response in the lung [49]. BALF from mice exposed to NJOY e-cig vapour for 2 weeks prior to challenging with Streptococcus pneumonia had significantly greater bacterial growth compared with their uninfected counterparts [47]. These findings suggest that exposure to e-cig aerosols may cause inflammation and may reduce the ability to clear a bacterial infection effectively. This dampened immunity had also been observed in a human study in which users self-reported their e-cig use [50].

E-cig plasma samples had a significant increase in MMP-9 levels. Similarly, a significant increase in MMP-9 levels was observed in induced sputum samples from e-cig users and cigarette smokers [13]. MMP-9 plays a role in breaking down the extracellular matrix in injured tissues. MMP-9 is involved in several diseases, including cigarette smoke-induced emphysema, coronary heart disease and COPD [51-53]. Our data suggest that exposure to e-cigs may induce acute pulmonary tissue injury. The increase of ICAM-1 in plasma samples of e-cig users suggests altered vascular permeability. This biomarker has also been associated with inflammation and progression of lung cancer [54]. 
RAGE plays a role in regulating cardiovascular and pulmonary disease progression in smokers [55, 56]. We observed lower levels of RAGE in the plasma of e-cig users compared with normal subjects. Although reports of serum levels of RAGE are variable in smokers [55], other studies have shown decreased levels [56-58].

Our results showed significant increases in plasma levels of desmosine in e-cig users compared with normal subjects. Plasma desmosine is a biomarker of elastin degradation, and is associated with cardiovascular risk and severity of COPD. Chronic cigarette smoking causes elastin degradation in the alveolar wall by proteases, leading to emphysema, cardiovascular risk and COPD [59,60]. E-cigs containing nicotine cause arterial stiffness and increases in blood pressure contributing to cardiovascular events [61]. Hence, long-term use may make e-cig users more susceptible to cardiovascular and pulmonary diseases [62, 63].

The levels of growth factors EGF, VEGF, $\beta$-NGF, PDGF-AA, SCF, HGF and PIGF were significantly increased in e-cig users compared with normal subjects, suggesting systemic effects of compensatory responses against other pro-inflammatory mediators. However, it is known that cigarette smoke alters the levels of VEGF, TGF- $\beta 1$ and FGFs [64-66]. This may be due to cigarette smoke-mediated alteration of other mediators directly/indirectly affecting endothelium/monocytes to alter growth factors. For example, cigarette smoking causes perturbation in VEGF-induced endothelial cell functions. Other growth factors, such as BDNF, BMP-2, TGF- $\alpha$ and bFGF/FGF2, showed no changes in e-cig users, suggesting that the e-cig effect is selective. Nevertheless, a long-term study is required to determine the mechanism of impact of e-cigs on angiogenesis and/or endothelium/monocyte regulation.

Pro-resolving anti-inflammatory mediators, such as resolvins $\mathrm{D}_{1}$ and $\mathrm{D}_{2}$, play a major role in dampening inflammation $[67,68]$. The levels of resolvins $\mathrm{D}_{1}$ and $\mathrm{D}_{2}$ were significantly decreased in plasma samples of e-cig users compared with normal subjects, suggesting downregulation of natural protecting mechanisms against inflammation and tissue repair. Thus, prolonged inflammation may lead to cardiovascular and pulmonary diseases.

Increased lung infiltration of macrophages, lymphocytes and neutrophils may occur as a result of chronic and subchronic e-cig use. A cross-sectional study suggested inflammasome complex proteins, caspase-1 and apoptosis-associated speck-like protein containing a caspase activation and recruitment domain, which promotes cellular pyroptosis, are elevated in the BALF of e-cig users [69]. Consistent with our observations in e-cig users, our study showed that pulmonary toxicity of e-cig aerosol exposure can induce inflammation, reduce/dampen host defence and protease-mediated lung injury. In a study correlating e-cig use with chronic bronchitis, the risk of disease symptoms (odds ratio) was increased approximately twofold in current and past e-cig users, and the risk was also increased with the frequency of vaping [70].

In summary, our study showed that e-cig users have increased levels of systemic biomarkers of inflammation, such as IL-1 $\beta$, IL-6, IL-8, IL-13, IFN- $\gamma$, RAGE and MMP-9. Levels of ICAM-1, a vascular biomarker, were also increased. Levels of desmosine, a biomarker for elastin breakdown, were also increased, suggesting extracellular matrix breakdown occurs rapidly in e-cig vapour-exposed lung tissue. Furthermore, levels of growth factors, such as EGF, VEGF, $\beta$-NGF, PDGF-AA, SCF, HGF and PIGF, were significantly increased. In addition, pro-resolving lipid mediators resolvin $\mathrm{D}_{1}$ and resolvin $\mathrm{D}_{2}$ were at low levels in e-cig users, indicating compromised anti-inflammatory, immunity and tissue repair mechanisms. The oxidative stress biomarker 8 -isoprostane, oxidative DNA damage biomarker 8-oxo-dG and fatty acid oxidation product triglycerides were also increased in e-cig users, suggesting e-cig vaping produces oxidative stress leading to damage in organ systems.

It is important to highlight that the levels of pro-inflammatory mediator IL-1 $\beta$ were increased by vaping across the biological fluids, whereas other inflammatory mediators (IL-6, IL-8 and IL-13), endothelial dysfunction (ICAM-1) and extracellular matrix breakdown and remodelling products (desmosine and MMP-9) were increased only in plasma, and oxidative stress biomarkers in urine of e-cig users. On the other hand, pro-resolving lipid mediators (resolvin $\mathrm{D}_{1}$ and $\mathrm{D}_{2}$ ) were decreased in other biological fluids in response to e-cig vaping. No correlation was found between biomarker levels when compared with each other in various biofluids.

\section{Conclusions}

This study suggests that e-cig use adversely affects oxidative stress, inflammatory responses and induces tissue remodelling. Prolonged activation of such mediators may lead to the progression of cardiovascular and pulmonary diseases. Some of the identified mediators across various biological fluids may be considered as hallmark noninvasive biomarkers for e-cig vaping. These biomarkers can be used for the assessment of e-cig vaping-associated lung injuries, and for regulatory and diagnostic aspects of vaping in humans.

Acknowledgements: We thank our participants and our research assistants and nurses, including Janice Gerloff (University of Rochester Medical Center, Rochester, NY, USA), for initial recruitment of subjects.

Author contributions: K.P. Singh, G. Lawyer, T. Muthumalage, N.A. Khan and I. Rahman conceived and designed the experiments, and wrote and edited the manuscript. I. Rahman obtained research funding, and designed the study and 
experimental plans/assays. N.A. Khan and I. Rahman recruited the volunteers. G. Lawyer, D. Ye, K.P. Maremanda and S.R. McDonough performed the experiments and analysed data. S. McIntosh designed the vaping surveys. All authors contributed to manuscript preparation and approved the final version before the submission.

Support statement: This work was supported in part by National Institutes of Health (NIH) grants 1R01HL135613 and 1R01HL085613, and the Food and Drug Administration (FDA) Center for Tobacco Products (CTP). In addition, it was in part supported by the National Cancer Institute of the NIH and the FDA CTP under award U54CA228110. The content is solely the responsibility of the authors and does not necessarily represent the official views of the NIH or the FDA. Funding information for this article has been deposited with the Crossref Funder Registry.

Conflict of interest: None declared.

\section{References}

1 Grana R, Benowitz N, Glantz SA. E-cigarettes: a scientific review. Circulation 2014; 129: 1972-1986.

2 Goniewicz ML, Knysak J, Gawron M, et al. Levels of selected carcinogens and toxicants in vapour from electronic cigarettes. Tob Control 2014; 23: 133-139.

3 Samburova V, Bhattarai C, Strickland M, et al. Aldehydes in exhaled breath during e-cigarette vaping: pilot study results. Toxics 2018; 6: E46.

4 Kaur G, Muthumalage T, Rahman I. Mechanisms of toxicity and biomarkers of flavoring and flavor enhancing chemicals in emerging tobacco and non-tobacco products. Toxicol Lett 2018; 288: 143-155.

5 Vansickel AR, Eissenberg T. Electronic cigarettes: effective nicotine delivery after acute administration. Nicotine Tob Res 2013; 15: 267-270.

6 Nides MA, Leischow SJ, Bhatter M, et al. Nicotine blood levels and short-term smoking reduction with an electronic nicotine delivery system. Am J Health Behav 2014; 38: 265-274.

7 Yan XS, D'Ruiz C. Effects of using electronic cigarettes on nicotine delivery and cardiovascular function in comparison with regular cigarettes. Regul Toxicol Pharmacol 2015; 71: 24-34.

8 Vlachopoulos C, Ioakeimidis N, Abdelrasoul M, et al. Electronic cigarette smoking increases aortic stiffness and blood pressure in young smokers. J Am Coll Cardiol 2016; 67: 2802-2803.

9 Qasim H, Karim ZA, Rivera JO, et al. Impact of electronic cigarettes on the cardiovascular system. J Am Heart Assoc 2017; 6: e006353.

10 Carnevale R, Sciarretta S, Violi F, et al. Acute impact of tobacco vs electronic cigarette smoking on oxidative stress and vascular function. Chest 2016; 150: 606-612.

11 Higashi Y, Noma K, Yoshizumi M, et al. Endothelial function and oxidative stress in cardiovascular diseases. Circ J 2009; 73: 411-418.

12 Antoniewicz L, Bosson JA, Kuhl J, et al. Electronic cigarettes increase endothelial progenitor cells in the blood of healthy volunteers. Atherosclerosis 2016; 255: 179-185.

13 Reidel B, Radicioni G, Clapp PW, et al. E-cigarette use causes a unique innate immune response in the lung, involving increased neutrophilic activation and altered mucin secretion. Am J Respir Crit Care Med 2018; 197: 492-501.

14 Meo SA, Ansary MA, Barayan FR, et al. Electronic cigarettes: impact on lung function and fractional exhaled nitric oxide among healthy adults. Am J Mens Health 2019; 13: 1557988318806073.

15 Shields PG, Berman M, Brasky TM, et al. A review of pulmonary toxicity of electronic cigarettes in the context of smoking: a focus on inflammation. Cancer Epidemiol Biomarkers Prev 2017; 26: 1175-1191.

16 Canistro D, Vivarelli F, Cirillo S, et al. E-cigarettes induce toxicological effects that can raise the cancer risk. Sci Rep 2017; 7: 2028.

17 Clapp PW, Jaspers I. Electronic cigarettes: their constituents and potential links to asthma. Curr Allergy Asthma Rep 2017; 17: 79

18 Chapman DG, Casey DT, Ather JL, et al. The effect of flavored e-cigarettes on murine allergic airways disease. Sci Rep 2019; 9: 13671.

19 Liu J, Liang Q, Frost-Pineda K, et al. Relationship between biomarkers of cigarette smoke exposure and biomarkers of inflammation, oxidative stress, and platelet activation in adult cigarette smokers. Cancer Epidemiol Biomarkers Prev 2011; 20: 1760-1769.

20 Shiels MS, Katki HA, Freedman ND, et al. Cigarette smoking and variations in systemic immune and inflammation markers. J Natl Cancer Inst 2014; 106: dju294.

21 Garcia-Rio F, Miravitlles M, Soriano JB, et al. Systemic inflammation in chronic obstructive pulmonary disease: a population-based study. Respir Res 2010; 11: 63.

22 Selvarajah S, Todd I, Tighe PJ, et al. Multiple circulating cytokines are coelevated in chronic obstructive pulmonary disease. Mediators Inflamm 2016; 2016: 3604842.

23 Rahman I, Morrison D, Donaldson K, et al. Systemic oxidative stress in asthma, COPD, and smokers. Am J Respir Crit Care Med 1996; 154: 1055-1060.

24 Repine JE, Bast A, Lankhorst I. Oxidative stress in chronic obstructive pulmonary disease. Am J Respir Crit Care Med 1997; 156: 341-357.

25 Morrow JD, Frei B, Longmire AW, et al. Increase in circulating products of lipid peroxidation ( $\mathrm{F}_{2}$-isoprostanes) in smokers. Smoking as a cause of oxidative damage. N Engl J Med 1995; 332: 1198-1203.

26 Pizzino G, Irrera N, Cucinotta M, et al. Oxidative stress: harms and benefits for human health. Oxid Med Cell Longev 2017; 2017: 8416763.

27 Christiani DC. Vaping-induced lung injury. N Engl J Med 2019; in press [https://doi.org/10.1056/NEJMe1912032].

28 Viswam D, Trotter S, Burge PS, et al. Respiratory failure caused by lipoid pneumonia from vaping e-cigarettes. BMJ Case Rep 2018; 2018: bcr-2018-224350.

29 Maddock SD, Cirulis MM, Callahan SJ, et al. Pulmonary lipid-laden macrophages and vaping. N Engl J Med 2019; 381: 1488-1489.

30 Schier JG, Meiman JG, Layden J, et al. Severe pulmonary disease associated with electronic-cigarette-product use interim guidance. MMWR Morb Mortal Wkly Rep 2019; 68: 787-790. 
31 Kennedy SM, Elwood RK, Wiggs BJ, et al. Increased airway mucosal permeability of smokers. Relationship to airway reactivity. Am Rev Respir Dis 1984; 129: 143-148.

32 Kinnula VL, Ilumets $\mathrm{H}$, Myllarniemi M, et al. 8-Isoprostane as a marker of oxidative stress in nonsymptomatic cigarette smokers and COPD. Eur Respir J 2007; 29: 51-55.

33 Montuschi P, Collins JV, Ciabattoni G, et al. Exhaled 8-isoprostane as an in vivo biomarker of lung oxidative stress in patients with COPD and healthy smokers. Am J Respir Crit Care Med 2000; 162: 1175-1177.

34 Czekala L, Simms L, Stevenson M, et al. Toxicological comparison of cigarette smoke and e-cigarette aerosol using a 3D in vitro human respiratory model. Regul Toxicol Pharmacol 2019; 103: 314-324.

35 Lee J, Taneja V, Vassallo R. Cigarette smoking and inflammation: cellular and molecular mechanisms. J Dent Res 2012; 91: 142-149.

36 van der Vaart H, Postma DS, Timens W, et al. Acute effects of cigarette smoke on inflammation and oxidative stress: a review. Thorax 2004; 59: 713-721.

37 Maggio M, Guralnik JM, Longo DL, et al. Interleukin-6 in aging and chronic disease: a magnificent pathway. J Gerontol A Biol Sci Med Sci 2006; 61: 575-584.

38 Tanaka T, Narazaki M, Kishimoto T. IL-6 in inflammation, immunity, and disease. Cold Spring Harb Perspect Biol 2014; 6: a016295.

39 Mukaida N. Pathophysiological roles of interleukin-8/CXCL8 in pulmonary diseases. Am J Physiol Lung Cell Mol Physiol 2003; 284: L566-L577.

40 Passalacqua G, Mincarini M, Colombo D, et al. IL-13 and idiopathic pulmonary fibrosis: Possible links and new therapeutic strategies. Pulm Pharmacol Ther 2017; 45: 95-100.

41 Joshi BH, Hogaboam C, Dover $\mathrm{P}$, et al. Role of interleukin-13 in cancer, pulmonary fibrosis, and other $\mathrm{T}_{\mathrm{H}} 2$-type diseases. Vitam Horm 2006; 74: 479-504.

42 Mokeem SA, Alasqah MN, Michelogiannakis $\mathrm{D}$, et al. Clinical and radiographic periodontal status and whole salivary cotinine, IL-1beta and IL-6 levels in cigarette- and waterpipe-smokers and E-cig users. Environ Toxicol Pharmacol 2018; 61: 38-43.

43 Rahimi S, Khosravi A, Aazami S, et al. Effect of smoking on cyanide, IL-2 and IFN- $\gamma$ levels in saliva of smokers and nonsmokers. Polish Ann Med 2018; 25: 203-206.

44 Scott A, Lugg ST, Aldridge K, et al. Pro-inflammatory effects of e-cigarette vapour condensate on human alveolar macrophages. Thorax 2018; 73: 1161-1169.

45 Bozier J, Rutting S, Xenaki D, et al. Heightened response to e-cigarettes in COPD. ERJ Open Res 2019; 5: 00192-2018.

46 Lerner CA, Sundar IK, Yao H, et al. Vapors produced by electronic cigarettes and e-juices with flavorings induce toxicity, oxidative stress, and inflammatory response in lung epithelial cells and in mouse lung. PLoS One 2015 10: e0116732.

47 Sussan TE, Gajghate S, Thimmulappa RK, et al. Exposure to electronic cigarettes impairs pulmonary anti-bacterial and anti-viral defenses in a mouse model. PLoS One 2015; 10: e0116861.

48 Husari A, Shihadeh A, Talih S, et al. Acute exposure to electronic and combustible cigarette aerosols: effects in an animal model and in human alveolar cells. Nicotine Tob Res 2016; 18: 613-619.

49 Hwang JH, Lyes $\mathrm{M}$, Sladewski $\mathrm{K}$, et al. Electronic cigarette inhalation alters innate immunity and airway cytokines while increasing the virulence of colonizing bacteria. J Mol Med 2016; 94: 667-679.

50 Li D, Sundar IK, McIntosh S, et al. Association of smoking and electronic cigarette use with wheezing and related respiratory symptoms in adults: cross-sectional results from the Population Assessment of Tobacco and Health (PATH) study, wave 2. Tob Control 2019; in press [https://doi.org/10.1136/tobaccocontrol-2018-054694].

51 Atkinson JJ, Lutey BA, Suzuki Y, et al. The role of matrix metalloproteinase- 9 in cigarette smoke-induced emphysema. Am J Respir Crit Care Med 2011; 183: 876-884.

52 Welsh P, Whincup PH, Papacosta O, et al. Serum matrix metalloproteinase-9 and coronary heart disease: a prospective study in middle-aged men. QJM 2008; 101: 785-791.

53 Gilowska I, Kasper L, Bogacz K, et al. Impact of matrix metalloproteinase 9 on COPD development in Polish patients: genetic polymorphism, protein level, and their relationship with lung function. Biomed Res Int 2018; 2018: 6417415.

54 Kotteas EA, Boulas P, Gkiozos I, et al. The intercellular cell adhesion molecule-1 (ICAM-1) in lung cancer: implications for disease progression and prognosis. Anticancer Res 2014; 34: 4665-4672.

55 Prasad K, Dhar I, Caspar-Bell G. Role of advanced glycation end products and its receptors in the pathogenesis of cigarette smoke-induced cardiovascular disease. Int J Angiol 2015; 24: 75-80.

56 Iwamoto $\mathrm{H}$, Gao J, Pulkkinen V, et al. Soluble receptor for advanced glycation end-products and progression of airway disease. BMC Pulm Med 2014; 14: 68.

57 Yokota C, Minematsu K, Tomii Y, et al. Low levels of plasma soluble receptor for advanced glycation end products are associated with severe leukoaraiosis in acute stroke patients. J Neurol Sci 2009; 287: 41-44.

58 Gopal P, Reynaert NL, Scheijen JL, et al. Association of plasma sRAGE, but not esRAGE with lung function impairment in COPD. Respir Res 2014; 15: 24.

59 Rabinovich RA, Miller BE, Wrobel K, et al. Circulating desmosine levels do not predict emphysema progression but are associated with cardiovascular risk and mortality in COPD. Eur Respir J 2016; 47: 1365-1373.

60 Luisetti M, Ma S, Iadarola P, et al. Desmosine as a biomarker of elastin degradation in COPD: current status and future directions. Eur Respir J 2008; 32: 1146-1157.

61 Chaumont $\mathrm{M}$, de Becker B, Zaher W, et al. Differential effects of e-cigarette on microvascular endothelial function, arterial stiffness and oxidative stress: a randomized crossover trial. Sci Rep 2018; 8: 10378.

62 Prochaska JJ. The public health consequences of e-cigarettes: a review by the National Academies of Sciences. A call for more research, a need for regulatory action. Addiction 2019; 114: 587-589.

63 Benowitz NL, Burbank AD. Cardiovascular toxicity of nicotine: implications for electronic cigarette use. Trends Cardiovasc Med 2016; 26: 515-523.

64 Mak JC, Chan-Yeung MM, Ho SP, et al. Elevated plasma TGF-betal levels in patients with chronic obstructive pulmonary disease. Respir Med 2009; 103: 1083-1089.

65 Schmidt-Lucke C, Belgore F, Reinhold D, et al. Soluble vascular endothelial growth factor, soluble VEGF receptor Flt-1 and endothelial function in healthy smokers. Int J Cardiol 2005; 100: 207-212.

66 Kamizono Y, Shiga Y, Suematsu Y, et al. Impact of cigarette smoking cessation on plasma alpha-klotho levels. Medicine 2018; 97: e11947. 
67 Serhan CN, Levy BD. Resolvins in inflammation: emergence of the pro-resolving superfamily of mediators. J Clin Invest 2018; 128: 2657-2669.

68 Croasdell A, Thatcher TH, Kottmann RM, et al. Resolvins attenuate inflammation and promote resolution in cigarette smoke-exposed human macrophages. Am J Physiol Lung Cell Mol Physiol 2015; 309: L888-L901.

69 Tsai M, Song MA, McAndrew C, et al. Electronic versus combustible cigarette effects on inflammasome component release into human lung. Am J Respir Crit Care Med 2019; 199: 922-925.

70 McConnell R, Barrington-Trimis JL, Wang $\mathrm{K}$, et al. Electronic cigarette use and respiratory symptoms in adolescents. Am J Respir Crit Care Med 2017; 195: 1043-1049. 